\title{
Parthenogenetic Activation of Mouse Oocytes by Strontium
}

\author{
Tomomi Yoshimizu1\#, Yayoi Obata ${ }^{1}$, John Carroll2 and \\ Tomohiro Kono'*
}

INODAI Research Institute, Tokyo University of Agriculture, Tokyo 156-0054, Japan and

${ }^{2}$ Department of Physiology, University College London, London, WCIE 6BT, UK.

\#Present address: Department of Cell Biology, Institute of Development,

Aging and Cancer, Tohoku University, Miyagi 980-8575, Japan

\begin{abstract}
The purposes of this study were to determine the optimal conditions for inducing parthenogenetic activation by strontium and to evaluate the developmental ability of the activated oocytes in mice. MII oocytes collected from B6CBF1 and $C D-1$ mice were cultured for 2-60 min in medium containing $1.7 \mathrm{mM}$ strontium. The proportion of oocytes activated increased in a time-dependent manner, with an apparent difference between the B6CBF1 and $C D-1$ strains in sensitivity to strontium. In oocytes derived from $B 6 C B F 1$ mice more than $90 \%$ of oocytes were activated by the treatment with strontium for $30 \mathrm{~min}$, whereas more than $80 \%$ of the oocytes from $C D-1$ mice were activated by the treatment for 5 min. The majority of oocytes formed the second polar body and a single pronucleus in both cases. Of the parthenogenetically activated 1-cell embryos with the haploid genome, around $39 \%$ developed to the blastocyst stage in the B6CBF1, and in contrast the developmental ability was low (11\%) in the CD-1 strain. The ability to develop to blastocysts was significantly improved in diploid parthenogenetic embryos of both the strains; $93 \%$ and $58 \%$ were developed to the blastocyst stage, respectively. After transfer of the diploid parthenogenetic embryos to recipient mice, $11 \%$ of the embryos developed to day 10 of gestation. The record of intracytoplasmic $\mathrm{Ca}^{2+}$ concentrations showed that the exposure of oocytes to medium containing $1.7 \mathrm{mM}$ strontium induced repetitive intracellular $\mathrm{Ca}^{2+}$ transients. These data clearly showed that strontium is a potent stimulus for inducing parthenogenetic activation and supporting in vitro and in vivo development in mouse oocytes.
\end{abstract}

Key words: Mouse oocyte, Parthenogenetic activation, Development, Strontium, Calcium transients.

Received: February 13, 1998

Accepted: March 11, 1998

*To whom correspondence should be addressed.
Oocytes are ovulated at the metaphase of the second meiosis and arrested at this stage of the cell cycle until fertilization. This process is regulated by the cytoplasmic maturation promoting factor (MPF), a complex of cyclin and p34cdc2, which is stabilized by cytostatic factor (CSF) [1-4]. Intracellular $\mathrm{Ca}^{2+}$ oscillations at fertilization, which are seen every 10-20 min and last for 3-4 h, are necessary for resumption of the second meiosis of the oocytes $[4,5]$. Artificial stimuli which cause intracytoplasmic $\mathrm{Ca}^{2+}$ increases can also induce oocyte activation. These artificial stimuli, such as exposure to ethanol, the $\mathrm{Ca}^{2+}$ ionophore $\mathrm{A} 23187$, or direct electric pulses, cause a single $\mathrm{Ca}^{2+}$ increase caused by the influx of exogenous $\mathrm{Ca}^{2+}[6]$, but strontium $\left(\mathrm{Sr}^{2+}\right)$ treatment mimics the repetitive $\mathrm{Ca}^{2+}$ oscillations seen at fertilization [7]. How $\mathrm{Sr}^{2+}$ causes the repetitive intracellular $\mathrm{Ca}^{2+}$ oscillations and meiotic resumption is unclear, but it is thought to provide a more efficient procedure for the induction of parthenogenetic activation in mouse oocytes, compared to the other stimuli which induce a single intracellular $\mathrm{Ca}^{2+}$ increase [5-9]. In addition, the difference between fertilization and parthenogenesis in cytoplasmic $\mathrm{Ca}^{2+}$ mobility may reflect the ability of the cytoplasm to support further development of embryos.

Although no precise experiment has been conducted, it is known that a wide range of concentrations of $\mathrm{Sr}^{2+}$, 1.7-10 mM, are effective in inducing intracellular $\mathrm{Ca}^{2+}$ oscillations [7-9] and oocyte activation in mice [8-10]. Incubation with medium containing $10 \mathrm{mM} \mathrm{Sr}^{2+}$ for 2 to $24 \mathrm{~h}$ was found to be quite efficient for oocyte activation; more than $90 \%$ of the oocytes formed the second polar body and pronucleus. The parthenogenetic 2-cell embryos, which had been diplodized by inhibition of the second polar body extrusion, developed to the blastocyst stage in the range of $73-87 \%$ in vitro, but few fetuses were obtained after transfer to recipients [9]. A 
possibility is that over stimulation by incubation with 10 $\mathrm{mM} \mathrm{Sr}{ }^{2+}$ medium for several hours has a detrimental effect on embryo development. Studies so far show that $\mathrm{Sr}^{2+}$ is certainly efficient in the induction of parthenogenetic activation in mouse oocytes, but optimal conditions for its use have not yet been established. We therefore examined the effects of duration of incubation with $\mathrm{Sr}^{2+}$ medium on the activation rate and the ability of the oocytes to develop to blastocysts and fetuses by using oocytes from B6CBF1 and CD-1 mice.

\section{Material and Methods}

\section{Oocyte collection}

$\mathrm{B} 6 \mathrm{CBF} 1(\mathrm{C} 57 \mathrm{BL} / 6 \mathrm{~N} \times \mathrm{CBA})$ and $\mathrm{CD}-1$ mice were used as oocyte donors. They were superovulated with injections of 5 iu of equine chorionic gonadotrophin (eCG; Peamex, Sankyo Ltd., Tokyo, Japan) and 5 iu of human chorionic gonadotrophin (hCG; Puberogen, Sankyo Ltd.) given $48 \mathrm{~h}$ apart. Oocytes at metaphase II were released from the oviducts $15 \mathrm{~h}$ after the $\mathrm{hCG}$ injection, and the cumulus cells were removed by digestion with 300 units/ml hyaluronidase in M2 medium [10]. After being washed with $\mathrm{M} 2$ medium several times, normal MII oocytes were selected and used for the experiments.

\section{Artificial activation}

To induce oocyte activation, $1.7 \mathrm{mM} \mathrm{SrCl}_{2}\left(\mathrm{Sr}^{2+}\right)$ was prepared in $\mathrm{Ca}^{2+-f r e e ~} \mathrm{M} 16$ medium [12] supplemented with $0.4 \%$ polyvinyl alcohol (PVA) and adjusted to 260 mOsm. Oocytes were cultured in a $50 \mu \mathrm{l}$ drop of $\mathrm{Sr}^{2+}$ medium for 2-60 $\mathrm{min}$ in an atmosphere of $5 \% \mathrm{CO}_{2}, 5 \%$ $\mathrm{O}_{2}$, and $90 \% \mathrm{~N}_{2}$ at $37^{\circ} \mathrm{C}$. The oocyte activation was evaluated by extrusion of the second polar body and formation of the pronuclei at $6 \mathrm{~h}$ after the treatment. Activated oocytes were classified into the following 4 types according to Webb et al. (1986) [13]: 1) oocytes which formed a second polar body and single pronucleus $(1 \mathrm{PN}+1 \mathrm{~PB}), 2)$ oocytes which formed two pronuclei without the second polar body (2PN), 3) oocytes immediately cleaved (IC), 4) oocytes with a single pronucleus without the second polar body (1PN), 5) oocytes which formed a second polar body and metaphase III chromosomes (MIII), and 6) others including degraded oocytes. The experiments were repeated 5 to 8 times.

\section{In vitro culture and embryo transfer}

After culture with $\mathrm{Sr}^{2+}$ medium for $1 \mathrm{~h}$, the oocytes were washed with M2 medium several times and cultured in a drop of M16 medium supplemented with $0.4 \%$ bovine serum albumin (BSA) and $100 \mu \mathrm{M}$ EDTA in an atmosphere of $5 \% \mathrm{CO}_{2}, 5 \% \mathrm{O}_{2}$, and $90 \% \mathrm{~N}_{2}$ at $37^{\circ} \mathrm{C}$. When diploid parthenogenetic embryos were produced, following the incubation with $\mathrm{Sr}^{2}+$ medium the oocytes were cultured in M16 medium containing $5 \mu \mathrm{g} / \mathrm{ml}$ cytochalasin $B(C B)$ to inhibit an extra-polar body extrusion and to induce the formation of two pronuclei.

The blastocysts derived from B6CBF1 diploid parthenogenetic embryos were transferred to the uterine horns of CD-1 females on day 3 of pseudopregnancy (2.5 days post coitum).

\section{Intracellular $\mathrm{Ca}^{2+}$ measurements}

To monitor intracellular $\mathrm{Ca}^{2+}$, oocytes were incubated for 15-20 min in $2 \mu \mathrm{M}$ fura 2 AM (Sigma) in M2 containing $0.002 \%$ pluronic. The zona pellucida was removed by a brief exposure to acidified Tyrode's medium, and the oocyte was transferred to a heated chamber $\left(37^{\circ} \mathrm{C}\right)$ on a Zeiss inverted fluorescent microscope. Fura-2 was excited at 340 and $380 \mathrm{~nm}$ and emitted light $>520 \mathrm{~nm}$ was collected. Images were acquired using a cooled CCD camera and were processed using lonvision software (Coventry, UK). Fluorescence records are presented as the ratio of fluorescence intensities acquired at 340 and $380 \mathrm{~nm}$.

\section{Evaluation of meiotic resumption}

To assess meiotic resumption after parthenogenetic activation, whole mount preparations of the oocytes were prepared at $0,15,30,60,90,120,180,240$ and 360 min after the beginning of the treatment. After being mounted on slide glass, oocytes were fixed with methanol 3: acetic acid 1 solution for 2 days and stained with $1 \%$ lacmoid.

\section{Stastical analysis}

Data were analyzed by $\chi^{2}$-test.

\section{Results}

\section{Proportion of activated oocytes}

In the first experiment, oocytes were incubated with $1.7 \mathrm{mM} \mathrm{Sr}^{2+}$ medium for 2-60 min to assess the optimal time for the induction of parthenogenetic activation. The activated oocytes were classified into 4 types (Table 1). The proportion of activated B6CBF1 mouse oocytes, which was estimated by extrusion of the second polar body, was increased with the time of incubation, and $93 \%$ of the oocytes were activated when incubated for 30 and $60 \mathrm{~min}$. The majority of the activated oocytes extruded the second polar body and formed a single pronucleus. Of the oocytes incubated for $30 \mathrm{~min}, 15 \%$ 
were arrested at MIII, where the oocytes extruded the second polar body but failed to form a pronucleus. In the 60 min cultures, the proportion of MIII oocytes was significantly $(p<0.05)$ decreased to $4 \%$, and the oocytes that formed two pronuclei without the second polar body extrusion was significantly $(p<0.05)$ increased to $12 \%$. The oocytes from CD-1 mice were much more sensitive to $\mathrm{Sr}^{2+}$. When these oocytes were incubated with 1.7 $\mathrm{mM} \mathrm{Sr}{ }^{2+}$ medium for only $5 \mathrm{~min}, 81 \%$ of the oocytes were activated (Table 2). Prolongation of the incubation time did not increase the proportion of oocytes activated, of which $69-73 \%$ formed the second polar body and a single pronucleus.

\section{Development of parthenogenetic embryos}

To assess the ability to develop to blastocysts, the parthenogenetic 1PN + 1PB 1-cell embryos were cul- tured for 4 days in vitro. The developmental ability of the embryos from B6CBF1 oocytes was improved with a prolongation of incubation time with $\mathrm{Sr}^{2+}$ medium. The highest proportion of the embryos which developed to blastocyst stage was $39 \%$ in the 60 min incubation. In contrast in the CD-1, $11 \%$ of blastocysts were obtained by $15 \mathrm{~min}$ incubation, but the proportion tended to be decreased by prolonged duration. The developmental ability of the parthenogenetic embryos was significantly enhanced when the second polar body extrusion was inhibited with cytochalasin B. In the diploid parthenogenetic embryos, 93\% (B6CBF1) and 58\% (CD-1) developed to blastocysts. These rate of developmental are similar to those of fertilized embryos studied in our laboratory.

It is known that parthenogenetic embryos produced by other artificial stimuli and gynogenetic embryos pro-

Table 1. Parthenogentic activation of mouse oocytes by strontium

\begin{tabular}{|c|c|c|c|c|c|c|c|c|}
\hline \multirow{2}{*}{$\begin{array}{l}\text { Exposure time } \\
(\text { min })\end{array}$} & \multirow{2}{*}{$\begin{array}{l}\text { No. of oocytes } \\
\text { examined }\end{array}$} & \multirow{2}{*}{$\begin{array}{l}\text { No. of oocytes } \\
\text { activated (\%) }\end{array}$} & \multicolumn{5}{|c|}{ Types of activated oocytes (\%)\# } & \multirow{2}{*}{ Abnomal } \\
\hline & & & $1 \mathrm{PN}+1 \mathrm{~PB}$ & $2 \mathrm{PN}$ & IC & 1PN & M III & \\
\hline \multicolumn{9}{|l|}{ B6CBF1 } \\
\hline 0 & 109 & $4(4)$ & $2(18)$ & 1 & 0 & 0 & 1 & 1 \\
\hline 5 & 103 & $45(44)^{* * * *}$ & $28(27)^{* * * *}$ & 0 & 0 & 0 & $16^{* * * *}$ & 1 \\
\hline 15 & 103 & $70(68)^{*}$ & $59(57)^{* * * *}$ & 1 & 0 & 0 & 9 & 3 \\
\hline 30 & 102 & 95 (93) & $76(75)$ & 3 & 0 & 1 & 15 & 1 \\
\hline 60 & 107 & $99(93)$ & $83(78)$ & $12^{* *}$ & 0 & 0 & $4^{* *}$ & 2 \\
\hline \multicolumn{9}{|l|}{ CD-1 } \\
\hline 0 & 101 & $33(33)$ & $9(9)$ & 0 & 1 & 0 & 21 & 1 \\
\hline 2 & 100 & $30(30)^{* * * *}$ & $20(20)^{*}$ & 0 & 0 & 0 & $9^{* *}$ & 5 \\
\hline 5 & 115 & $94(81)^{* * * *}$ & $85(74)^{* * * *}$ & 0 & 1 & 0 & 8 & $1^{* * *}$ \\
\hline 15 & 142 & $114(80)$ & $99(70)$ & $4^{*}$ & 4 & 1 & 5 & $8^{* *}$ \\
\hline 30 & 129 & $101(78)$ & $89(69)$ & 4 & 5 & 0 & 1 & 6 \\
\hline 60 & 132 & $110(83)$ & $97(73)$ & 7 & 4 & 0 & 1 & 13 \\
\hline
\end{tabular}

\#See text for the abbreviations. Values with asterisks are significantly different from the preceding value; ${ }^{*} \mathrm{p}<0.1,{ }^{* *} \mathrm{p}<0.05,{ }^{* * *} \mathrm{p}<0.01,{ }^{* * *} \mathrm{p}<0.001$.

Table 2. In vitro development of parthenogentic mouse embryos activated by strontium

\begin{tabular}{ccc}
\hline $\begin{array}{c}\text { Exposure time } \\
(\mathrm{min})\end{array}$ & \multicolumn{2}{c}{ No. of activated oocytes developed to blastocysts/examined (\%) } \\
\cline { 2 - 3 } & B6CBF1 & CD-1 \\
Haploid parthenotes & & \\
5 & $2 / 28(7)$ & $3 / 85(4)$ \\
15 & $11 / 59(19)$ & $11 / 99(11)^{*}$ \\
30 & $28 / 76(37)^{*}$ & $10 / 89(9)$ \\
60 & $32 / 83(39)$ & $6 / 97(6)$ \\
Diploid parthenotes\# & & \\
60 & $102 / 110(93)^{* * * *}$ & $72 / 124(58)^{* * * *}$ \\
\hline
\end{tabular}

\#Diploid parthenogentic embryos were produced by inhibition of the second polar body extrusion. Values with asterisks are significantly different from the preceding value in each strain; ${ }^{*} p<0.1,{ }^{* * * *} p<0.001$. 
duced by pronuclear transfer are able to develop by day 10 of gestation, 20 - to 25 -somite fetuses. To reveal this ability in the parthenogenetic embryos activated by $\mathrm{Sr}^{2+}$ medium, a total of 104 diploid blastocysts derived from

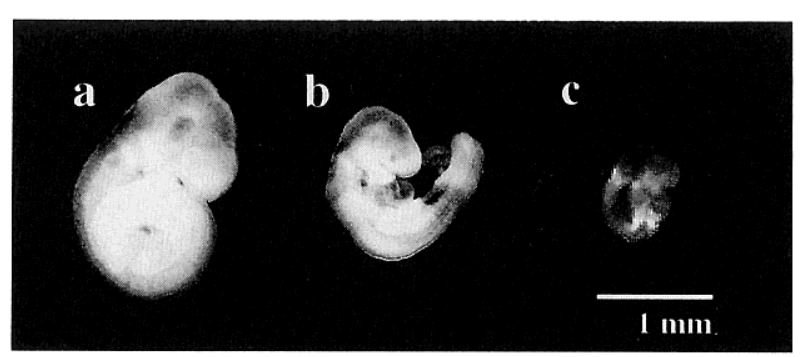

Fig. 1. Day 9.5 mouse fetuses, about 30 somites (a), 20 somites (b) and 10 somites (c), derived from oocytes activated parthenogenetically by strontium. the B6CBF1 oocytes were transferred into 10 pseudopregnant females. Of the transferred embryos, $11 \%$ developed to day 10 of gestation showing the various stages of development; and they had 8 to 30 somites (Table 3, Fig. 1).

\section{Cytokinetic changes in activated oocytes}

The progress of meiosis after the parthenogenetic activation was examined in B6CBF1 oocytes incubated with $1.7 \mathrm{mM} \mathrm{Sr}^{2+}$ medium for $60 \mathrm{~min}$ (Table 4). The transit from metaphase to anaphase of the second meiotic division started $15 \mathrm{~min}$ after the beginning of culture with $1.7 \mathrm{mM} \mathrm{Sr}^{2+}$ medium. At $30 \mathrm{~min}$ after the start of incubation, $86 \%$ of the oocytes were at the telophase of the second meiosis. The oocytes started to extrude the second polar body at 90 min after the treatment, when chromosomes were at the telophase stage, and within $120 \mathrm{~min}$ after the activation, most of the oocytes com-

Table 3. Postimplanation development of parthenogenetic mouse embryos activated by strontium

\begin{tabular}{|c|c|c|c|c|}
\hline No. recipients & $\begin{array}{l}\text { No. of embryos } \\
\text { transferred }\end{array}$ & $\begin{array}{l}\text { No. of embryos } \\
\text { implanted }\end{array}$ & No. of fetuses & \\
\hline 1 & 12 & 10 & 0 & \\
\hline 2 & 16 & 8 & 1 & 10 somites; 1 \\
\hline 3 & 14 & 8 & 0 & \\
\hline 4 & 11 & 11 & 0 & \\
\hline 5 & 20 & 16 & 0 & \\
\hline 6 & 13 & 10 & 3 & 25 somites; $1,10-15$ somites; 1,10 somites; 1 \\
\hline 7 & 8 & 7 & 5 & $20-25$ somites; $1,10-15$ somites; $2,8-10$ somites; 2 \\
\hline 8 & 13 & 12 & 1 & 10 somites; 1 \\
\hline 9 & 14 & 13 & 1 & 23 somites; 1 \\
\hline 10 & 11 & 9 & 4 & $25-30$ somites; 1,25 somites; $1,8-10$ somites; 2 \\
\hline Total 10 & 132 & $\begin{array}{l}104 \\
78.8 \%\end{array}$ & $\begin{array}{l}15 \\
11.4 \%\end{array}$ & \\
\hline
\end{tabular}

Table 4. Meiotic progress in mouse oocytes activated by strontium

\begin{tabular}{|c|c|c|c|c|c|c|c|}
\hline \multirow{2}{*}{$\begin{array}{l}\text { Time after\# } \\
\text { activation } \\
\text { (min) }\end{array}$} & \multirow{2}{*}{$\begin{array}{c}\text { No. of } \\
\text { oocytes } \\
\text { examined }\end{array}$} & \multicolumn{6}{|c|}{ Meiotic stages } \\
\hline & & MII & AnaII & TeloII & Telo/Retic ${ }^{1}$ & Reticular $^{2}$ & Pronuclear \\
\hline 0 & 15 & 15 & 0 & 0 & 0 & 0 & 0 \\
\hline 15 & 25 & 23 & 2 & 0 & 0 & 0 & 0 \\
\hline 30 & 29 & 1 & 3 & 25 & 0 & 0 & 0 \\
\hline 60 & 29 & 1 & 0 & 28 & 0 & 0 & 0 \\
\hline 90 & 21 & 0 & 0 & 13 & 8 & 0 & 0 \\
\hline 120 & 24 & 0 & 0 & 4 & 20 & 0 & 0 \\
\hline 180 & 21 & 0 & 0 & 0 & 0 & 21 & 0 \\
\hline 240 & 20 & 0 & 0 & 0 & 0 & 0 & 20 \\
\hline 360 & 21 & 0 & 0 & 0 & 0 & 0 & 21 \\
\hline
\end{tabular}

\# Oocytes were incubated with medium containing $1.7 \mathrm{mM} \mathrm{Sr}^{2+}$ for $60 \mathrm{~min}$. 1. Telo/Retic; oocytes that have the extruded second polar body, but the chromosomes are still condensed. 2. Reticular; oocytes that contain reticular stage nucleus. 


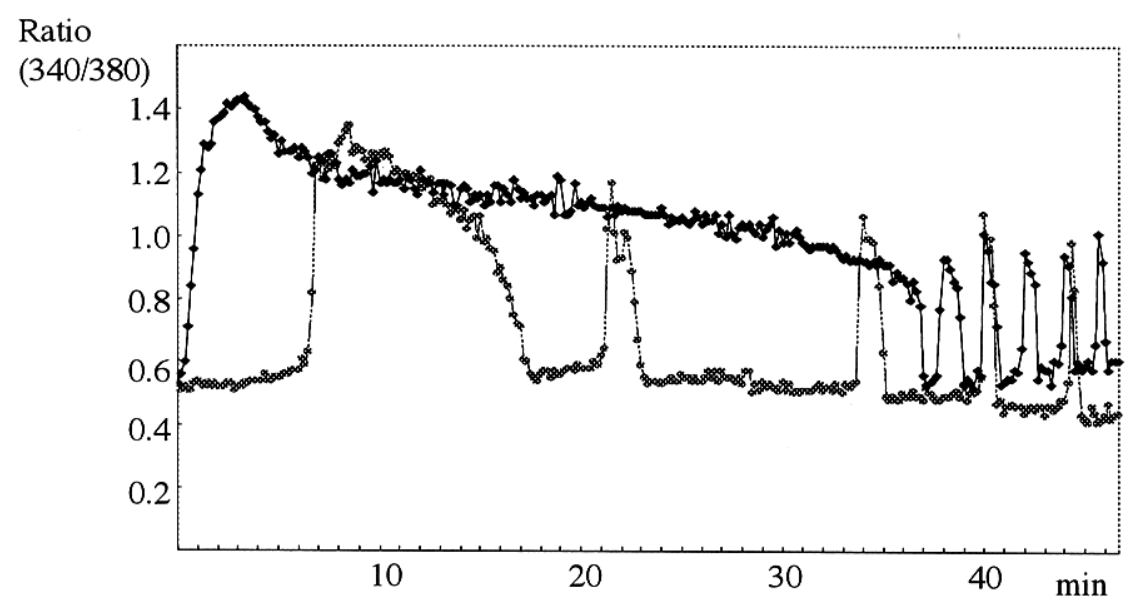

Fig. 2. Records of intracellular $\mathrm{Ca}^{2+}$ oscillations in the high- $(\diamond-\downarrow)$ and low-sensitive (1) mouse oocytes exposed to the strontium medium.

pleted the second polar body extrusion. Nuclear decondensation was seen from 180 min after activation, in which the nucleus was in the reticular stage, and pronuclei with nucleoli were observed at 240 min after activation.

\section{Intracellular $\mathrm{Ca}^{2+}$ oscillations}

To confirm whether parthenogenetic oocyte activation with $\mathrm{Sr}^{2+}$ medium is induced by an increase in intracellular $\mathrm{Ca}^{2+}$, intracellular $\mathrm{Ca}^{2+}$ levels were recorded. Intracellular $\mathrm{Ca}^{2+}$ oscillations were seen in all cases examined $(n=6)$. Figure 2 shows two records which represent high-sensitive and low-sensitive oocytes. The oocytes that sensitively responded to $\mathrm{Sr}^{2+}$ had a long first intracellular $\mathrm{Ca}^{2+}$ transient which lasted for $30 \mathrm{~min}$, starting $1 \mathrm{~min}$ after exposure to $\mathrm{Sr}^{2}+$ medium. In these cases the first transient was followed by repeatad $\mathrm{Ca}^{2+}$ transients at 5-10 min intervals. The low-sensitive oocytes showed the first $\mathrm{Ca}^{2+}$ transient from $7 \mathrm{~min}$ after the exposure; it lasted for $10 \mathrm{~min}$ and was followed by repetitive $\mathrm{Ca}^{2+}$ transients at 10-15 min intervals.

\section{Discussion}

The present study has confirmed that $\mathrm{Sr}^{2+}$ medium can be used to induce parthenogenetic activation and intracellular $\mathrm{Ca}^{2+}$ oscillations in mouse oocytes, as reported previously $[7,8,14]$. The analysis of cytokinesis in oocytes activated by $\mathrm{Sr}^{2+}$ showed that the majority of oocytes formed a single haploid nucleus after the extrusion of the second polar body [8-10]. The present study showed that the sequential events after incubation with
$\mathrm{Sr}^{2+}$ medium occurred in similarly to those seen in the fertilized oocytes, in which the second polar body extrusion occurred 90-120 min after the beginning of incubation in $\mathrm{Sr}^{2+}$ medium and formed a pronucleus 2.5$3 \mathrm{~h}$ later. Nevertheless, the sensitivity of oocytes to $\mathrm{Sr}^{2+}$ is apparently different in the two mouse strains, B6CBF1 and CD-1. A 30-min incubation in $\mathrm{Sr}^{2+} \mathrm{me}-$ dium was required to induce parthenogenetic activation in the oocytes from B6CBF1 mice. In contrast, the oocytes from CD-1 mice sensitively responded to $\mathrm{Sr}^{2+}$ medium and were activated by 5 -min incubation. The reason for the difference between the two strains in sensitivity to $\mathrm{Sr}^{2+}$ medium is unclear, but it has been reported that resistance to electric stimuli of the two strains also differs [15]. There is no evidence that $\mathrm{Sr}^{2+}$ is a potent stimulus for oocyte activation in other species; at least bovine oocytes do not respond to $\mathrm{Sr}^{2+}$ medium (unpublished data).

The ability of $\mathrm{Sr}^{2+}$ to activate mouse oocytes was first reported by Fraser [16], who showed that oocytes incubated in $\mathrm{Sr}^{2+}$ medium formed a second polar body and pronuclei. Kline and Kline [7] demonstrated that $\mathrm{Sr}^{2+}$ is able to mimic the repetitive intracellular $\mathrm{Ca}^{2+}$ transients seen at fertilization. Moreover, it is known that the proportion of aneuploidy in embryos activated with $\mathrm{Sr}^{2+}$ medium is significantly lower than in embryos activated by brief exposure to ethanol medium [10]. In view of these reports, $\mathrm{Sr}^{2+}$ is thought to be an efficient stimulus for inducing parthenogenetic activation in mouse oocytes, but the developmental ability of the oocytes has not been closely studied. A recent report by BosMikich et al. [9] showed that oocytes incubated in $\mathrm{Sr}^{2+}$ 
medium for 2-24 $\mathrm{h}$ developed to the blastocyst stage in vitro and the number of inner cell mass cells of blastocysts was significantly increased when oocytes were activated by the prolonged incubation in $\mathrm{Sr}^{2+}$ medium. This suggests that the intracellular $\mathrm{Ca}^{2+}$ oscillations play a role not only in the resumption of meiosis but also in the subsequent embryo development. But these embryos failed to develop to fetuses after transfer to recipient females except in a few cases. The present study on B6CBF1 oocytes clearly showed that oocytes activated with $\mathrm{Sr}^{2+}$ have the ability to develop in vitro and in vivo. The proportion of the activated oocytes developed to blastocysts was affected by the duration of incubation in $\mathrm{Sr}^{2}+$ medium. The developmental ability of oocytes incubated for short periods was poor, being areested until the 8-cell stage. As reported previously, the developmental ability of parthenogenetic 1-cell embryos was apparently enhanced by diplodization: $93 \%$ and $58 \%$ developed to blastocysts in B6CBF1 and CD1 embryos, respectively.

The development of parthenogenetic embryos after implantation is generally limited in mammals $[17,18]$. In mice, parthenogenetic and gynogenetic embryos are unable to develop beyond day 10 of gestation $[19,20]$. The proportion of embryos which developed to fetuses has varied among the reports and among the mouse species used. Gynogenetic mouse embryos which are produced by pronuclear exchange and contain only the maternal genome exhibit a slightly better ability to develop in vitro, but the post implantation development is not improved; only $10 \%$ of embryos develop to day 9.5 fetuses after transfer to recipient females [21]. In the reported by Bos-Mikich et al. [9], 60-70\% of the embryos transferred became successfully implanted but only $2 \%$ of the embryos were developed to day 10 of gestation. In the present study, regarding the developmental ability of the parthenogenetic embryos, $11 \%$ of the embryos transferred developed to day 10 of gestation. Our results revealed that diploid parthenogenetic embryos that are activated by $\mathrm{Sr}^{2+}$ medium are able to develop to day 10 of gestation, as can the gynogenetic embryos. These results suggest that insufficient stimulation with $\mathrm{Sr}^{2+}$ results in poor development of the parthenogenetic embryos in vitro, but over stimulation may have a detrimental effect on development, especially after implantation.

In conclusion, $\mathrm{Sr}^{2+}$ is a potent stimulus for inducing parthenogenetic activation in mouse oocytes, but the duration of incubation in $\mathrm{Sr}^{2+}$ medium affects activation of the oocytes and the subsequent development in vitro and in vivo.

\section{Acknowledgments}

This work was supported in part by grants-in-aid from the Ministry of Education, Science and Culture of Japan (07660383), The Japanese Society for the Promotion of Science (JSPS-RFTF9700905), the Ito Foundation, and the Association of Livestock Technology (Japan).

\section{References}

1) Kubiak, J.Z., Weber, M., Pennart, H., Winston, N.J. and Maro, B. (1993): The metaphase II arrest in mouse oocytes is controlled through microtubule-dependent destruction of cyclin $B$ in the presence of CSF. The EMBO Journal, 12, 3773-3778.

2) Choi, H., Aoki, F., Mori, M., Yamashita, M., Nagahama, Y. and Kohmoto, K. (1991): Activation of p34 ${ }^{\text {cdc2 }}$ protein kinese activity in meiotic and miotic cell cycle in mouse oocytes and embryos. Development, 113, 789-795.

3) Zernicka-Goetz, M., Ciemerych, M., Kubiak, J., Tarkowski, A. and Maro, B. (1995): Cytostatic factor inactivation is induced by a calcium-dependent mechanism present until the second cell cycle in fertilized but not in parthenogenetically activated mouse eggs. J. Cell Sci., 108, 469-474.

4) Whitaker, M. (1996): Control of meiotic arrest. Reviews of Reproduction 1, 127-135.

5) Swann, K. and Ozil, P. (1994): Dynamics of the calcium signal that triggers mammalian egg activation. Int. Rev. Cytol., 152, 182-222.

6) Whittingham, D.G. (1980): Parthenogenesisi in mammals. In: Oxford Reviews of Reproductive Biology (Finn, C.A., ed.), pp. 205-231, Oxford Univ. Press, London.

7) Kline, D. and Kline, J.T. (1992): Repetitive calcium transients and the role of calcium in exocytosis and cell cycle activation in the mouse egg. Dev. Biol., 149, 80-89.

8) Bos-Mikich, A., Swann, K. and Whittingham, D.G. (1995): Calcium oscillations and protein synthesis inhibition synergistically activate mouse oocytes. Mol. Reprod. Dev., 41, 84-90.

9) Bos-Mikich, A., Whittingham, D.G. and Jones, K.T. (1997): Meiotic and mitotic $\mathrm{Ca}^{2+}$ oscillations affect cell composition in resulting blastocysts. Dev. Biol., 182, 172-179.

10) O'Neill, G.T., Rolfe, L.R. and Kaufman, M.H. (1991): Developmental potential and chromosome constitution of strontium- induced mouse parthenogenones. Mol. Reprod. Dev., 30, 214-219.

11) Fulton, B. and Whittingham, D. (1978): Activation of mammalian oocytes by intracelluar injection of calcium. Nature, 273, 149-151.

12) Whittingham, D. (1971): Culture of mouse ova. J. 
Reprod. Fert. suppl., 7-12.

13) Webb, M., Howlett, S.K. and Maro, B. (1986): Parthenogenesis and cytoskeletal organization in ageing mouse eggs. J. Embryol. Exp. Morphol., 95, 131-145.

14) Kline, D. and Kline, J.T. (1992): Thapsigargin activates a calcium Influx pathway in the unfertilized mouse egg and suppresses repetitive calcium transients in the fertilized egg. J. Biol. Chem., 267, 1762417630.

15) Kono, T. and Tsunoda, Y. (1988): Effects of induction current and other factors on large-scale electrofusion for pronuclear transplantation of mouse eggs. Gamete Res., 19, 349-357.

16) Fraser, L. (1987): Strontium supports capacitation and the acrosome reaction in mouse sperm and rapidly activates mouse eggs. Gamete Res., 18, 363-374.

17) Monk, M. (1988): Genome imprinting. Genes and Dev.,
2, 921-925.

18) Surani, M.A., Kothary, R., Allen, N.D., Sigh, R.B., Fundele, R., Ferguson-smith, A.C. and Barton, S.C. (1990): Genome imprinting and development in the mouse. Development supplement, 89-98.

19) Barton, S.C., Adams, C.A., Norris, M.L. and Surani, M.A. (1985): Development of gynogenetic and parthenogenetic inner cell mass and trophectoderm tissues in reconstituted blastocysts in the mouse. J. Embryol. Exp. Morphol., 90, 267-285.

20) Kono, T., Obata, Y., Yoshimizu, T., Nakahara, T. and Carroll, J. (1996): Epigenetic modifications during oocyte growth correlates with extended parthenogenetic development in the mouse. Nature Genet., 13, 91-94.

21) Barton, S.C., Surani, M.A. and Norris, M.L. (1984): Role of paternal and maternal genomes in mouse development. Nature, 311, 374-376. 